\title{
Hotspot Decorations Map Plasmonic Patterns with the Resolution of Scanning Probe Techniques
}

\author{
V. K. Valev, ${ }^{1, *}$ A. V. Silhanek, ${ }^{2}$ Y. Jeyaram, ${ }^{2}$ D. Denkova, ${ }^{2}$ B. De Clercq, ${ }^{3}$ V. Petkov, ${ }^{4}$ X. Zheng, ${ }^{5}$ V. Volskiy, ${ }^{5}$ W. Gillijns, ${ }^{2}$ \\ G. A. E. Vandenbosch, ${ }^{5}$ O. A. Aktsipetrov, ${ }^{6}$ M. Ameloot,${ }^{2}$ V. V. Moshchalkov, ${ }^{2}$ and T. Verbiest ${ }^{1}$ \\ ${ }^{1}$ Molecular Electronics and Photonics, INPAC, Katholieke Universiteit Leuven, Celestijnenlaan 200 D, B-3001 Leuven, Belgium \\ ${ }^{2}$ Nanoscale Superconductivity and Magnetism \& Pulsed Fields Group, INPAC, Katholieke Universiteit Leuven, \\ Celestijnenlaan 200 D, B-3001 Leuven, Belgium \\ ${ }^{3}$ University Hasselt and transnational University Limburg, BIOMED, Diepenbeek, Belgium \\ ${ }^{4}$ Department of Metallurgy and Materials Engineering, Katholieke Universiteit Leuven, Kasteelpark Arenberg 44, B-3001, Belgium \\ ${ }^{5}$ ESAT-TELEMIC, Katholieke Universiteit Leuven, B-3001 Leuven, Belgium \\ ${ }^{6}$ Department of Physics, Moscow State University, 11992 Moscow, Russia
}

(Received 27 January 2011; revised manuscript received 23 March 2011; published 2 June 2011)

In high definition mapping of the plasmonic patterns on the surfaces of nanostructures, the diffraction limit of light remains an important obstacle. Here we demonstrate that this diffraction limit can be completely circumvented. We show that upon illuminating nanostructures made of nickel and palladium, the resulting surface-plasmon pattern is imprinted on the structures themselves; the hotspots (regions of local field enhancement) are decorated with overgrowths, allowing for their subsequent imaging with scanning-probe techniques. The resulting resolution of plasmon pattern imaging is correspondingly improved.

DOI: 10.1103/PhysRevLett.106.226803

PACS numbers: 73.20.Mf, 42.65.Ky, 78.67.Pt, 81.05.Xj

In the past ten years, a new class of materials has emerged and attracted a lot of attention due to its counterintuitive optical behavior and revolutionary potential applications-metamaterials. Metamaterials are specifically engineered materials for possessing unusual electromagnetic properties, which have been associated with negative refractive index [1], invisibility [2], light-based nanocircuits [3-5], etc. Most of these spectacular phenomena are based on surface-plasmon resonances-the property whereby, in metallic nanostructures, light can collectively excite surface electron waves. In metallic nanostructures, the imaging of surface-plasmon resonances provides a direct way to map the local electric fields that are responsible for the unusual electromagnetic properties of metamaterials. Recently, several techniques have been developed to achieve imaging resolution beyond that of the diffraction limit. For instance, cathodoluminescence [6] and electron energy loss spectroscopy [7] both offer the high resolution of a scanning electron microscope, though they can only be used in vacuum. An alternative can be found in scattering type, also known as apertureless, scanning near-field optical microscope [8]. This technique involves scanning a sharp probe, for instance an atomic force microscope tip, above an illuminated sample. As the probe enters the near field of the sample, a small fraction of light is scattered and is then collected. The technique can achieve resolution of $50 \mathrm{~nm}$ and better, providing information on both amplitude and phase $[9,10]$. The trade-off for this improved resolution is a significantly more complex experimental arrangement, whereby interaction between the scanning-probe tip and the particle being studied complicates the interpretation of the measured signal; moreover, for large sample areas and high-resolution imaging, very long scanning time is required. Generally speaking, while there are methods to go beyond the diffraction limit of light, they come at a considerable increase in both cost and complexity.

Here we show that, in measuring plasmonic patterns in nanostructures, the diffraction limit of light can be completely circumvented. We demonstrate that upon illuminating nanostructures made of nickel or palladium, the resulting surface-plasmon pattern is imprinted on the structures themselves. This imprinting is done through migrating material from the nanostructure itself to the hotspots, where it forms overgrowths. In this manner, the hotspots are effectively decorated, allowing for subsequent imaging with scanning electron microscopy (SEM) or atomic force microscopy (AFM). The imprinting method is quite unique, combining aspects of both imaging and writing [11] techniques. The combination offers a resolution on local field enhancements that can, in principle, be brought down to that of the AFM. Moreover, the technique is very user-friendly; the actual hotspot decoration, from the moment the sample is placed on the microscope to the moment it is removed typically takes 5 to $10 \mathrm{~min}$ for an area of $10 \times 10 \mu \mathrm{m}$. Implementation of the technique requires a standard confocal or second harmonic generation microscope.

Our samples consist of a periodic array $(3333 \times 3333$ elements) of $G$-shaped Ni nanostructures on $\mathrm{SiO}_{2} / \mathrm{Si}(001)$ substrate. The SEM picture in Fig. 1(a) shows the unit cell of that array, which contains four Gs-each rotated at $90^{\circ}$ with respect to its neighbors. An individual $G$ is $1 \mu \mathrm{m}$ in size, $25 \mathrm{~nm}$ thick, and the line is $200 \mathrm{~nm}$ wide. The SEM 

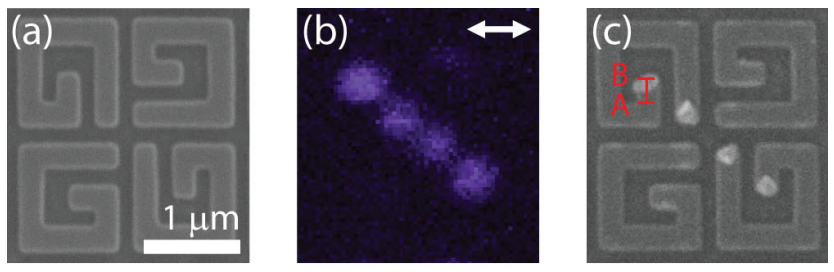

(d)

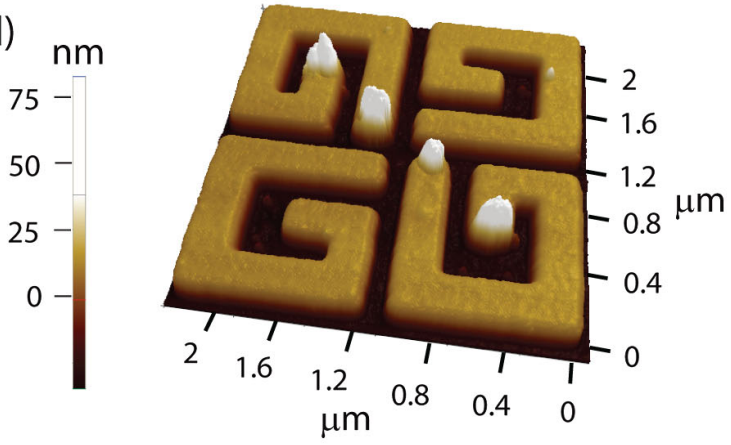

(e)

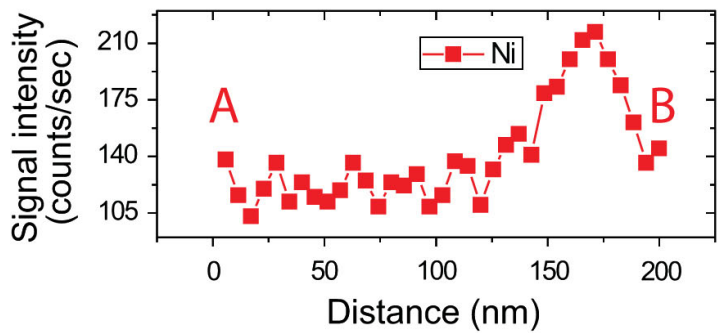

FIG. 1 (color online). The surface-plasmon pattern can be imprinted on the material. (a) Scanning electron microscopy (SEM) image of $G$-shaped nanostructures made of $\mathrm{Ni}$, before illumination. (b) Upon illumination with $800 \mathrm{~nm}$ light, second harmonic microscopy reveals localized field enhancements due to plasmons. The color-coded intensities increase from black through purple to pink and the white arrow indicates the direction of the incident light polarization. (c) SEM images after illumination show that the plasmonic pattern has been imprinted on the structures. (d) AFM images confirm that this imprint (plasmonic hotspot decorations) is of structural nature rather than electron specific. (e) Energy dispersive spectroscopy (EDS) shows that the decorations in (d) are made of $\mathrm{Ni}$, most likely oxidized. The EDS was performed along the red segment $A, B$ in (c).

picture in Fig. 1(a) shows the structures before illuminating them. Illumination is done by a Ti:sapphire femtosecond pulsed laser at a wavelength of $800 \mathrm{~nm}$ and with a very small average power on the sample $(0.98 \mathrm{~mW})$. The resulting local field enhancements in the nanostructures can be visualized with second harmonic generation (SHG) microscopy [12], as shown in Fig. 1(b). Four hotspots are revealed, which correspond to localized sources of SHG. The latter are due to maxima in the local plasmonic currents $[13,14]$, which have also been observed in similarly structured samples made of gold [15]. Indeed, SHG microscopy has recently been demonstrated as a valuable tool for studying plasmonic patterns in nanostructures [12]. Nevertheless, as can be seen in Fig. 1(b), the hotspots are relatively large.
In Fig. 1(c), another SEM image of the unit cell is displayed; the image was taken after illumination. At variance with the image in Fig. 1(a), four white spots are now present, perfectly matching the pattern of the plasmons in Fig. 1(b). These white spots result from an imprint of the plasmonic pattern on the structures, most likely under the influence of the intense local electric fields in the hotspots, as will be discussed later on in this Letter. It is difficult to judge from the SEM micrographs alone what these white spots really are. For instance, they could be melted Ni. In order to verify that these white spots are indeed related to the structure of the samples, AFM was performed; see Fig. 1(d). Surprisingly, the AFM results reveal very high plasmonic hotspot decorations, corresponding to the SEM white spots. What is this extra material? While the lift-off procedure does remove most of the resist, there is always a small quantity that remains on the sample, especially in the corners and on the edges. Perhaps some of this remaining resist found its way into the plasmonic hotspots. Other possible sources of material are the substrate, the Ni structures, the atmosphere, and accidental contacts with chemicals during sample preparation and handling. In order to understand the origin of the extra material, we should examine its composition. Consequently, we performed an energy dispersive spectroscopy (EDS) scan along the red segment $[A, B]$ in Fig. 1(c).

As the scan progresses along the $\mathrm{Ni}$ nanostructure and crosses over the white spot, the EDS curve shows an increase of the amount of Ni; see Fig. 1(e). Similarly, an increase in oxygen was observed. Therefore, the plasmonic hotspot decorations are most likely made of atmosphereoxidized Ni; i.e., they originate in the Ni $G$-shaped nanostructures themselves and indeed constitute an imprint. But is this really an imprint of the local field enhancements?

Figure 2(a) shows the result of a numerical simulation with MAGMAS that maps the currents at the surface of the nanostructures. MAGMAS is a numerical software tool originally developed at the K.U. Leuven for electromagnetic problems in the microwave and millimeter wave frequency bands $[16,17]$. It has recently been extended to plasmonics. In Fig. 2(a), the four hotspots along the main diagonal are clearly visible within the full-line white circles. Additionally, two off-diagonal field enhancements can be seen within the dashed-line white circles. These field enhancements are clearly not visible in Fig. 1, most likely because they are less intense. Indeed, upon increasing the laser power from $0.98 \mathrm{~mW}$ [in Fig. 2(b)] to $1.23 \mathrm{~mW}$ [in Fig. 2(c)], the two off-diagonal SHG hotspots do become visible. After this illumination, the resulting hotspot decorations are presented in Fig. 2(d). The figure clearly shows two off-diagonal plasmonic hotspot decorations, situated at the edge of the nanostructures; they are both approximately $175 \mathrm{~nm}$ long and $100 \mathrm{~nm}$ wide. These dimensions pinpoint the location of the field enhancements in our samples in perfect agreement with the numerical simulation. 
(a)

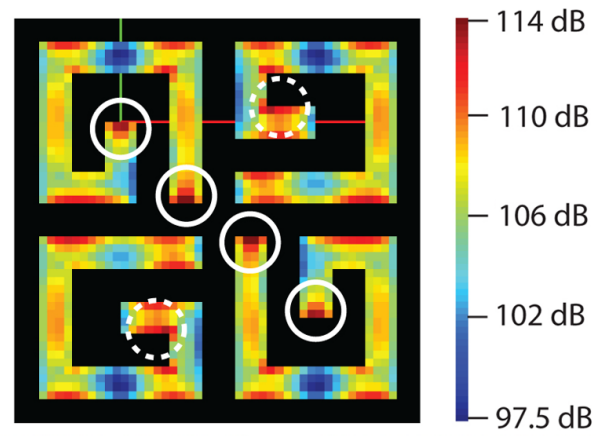

(b)

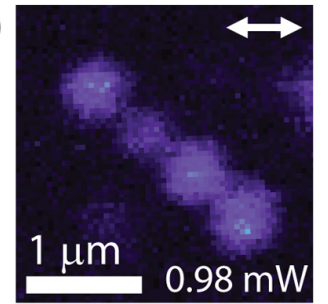

(c)
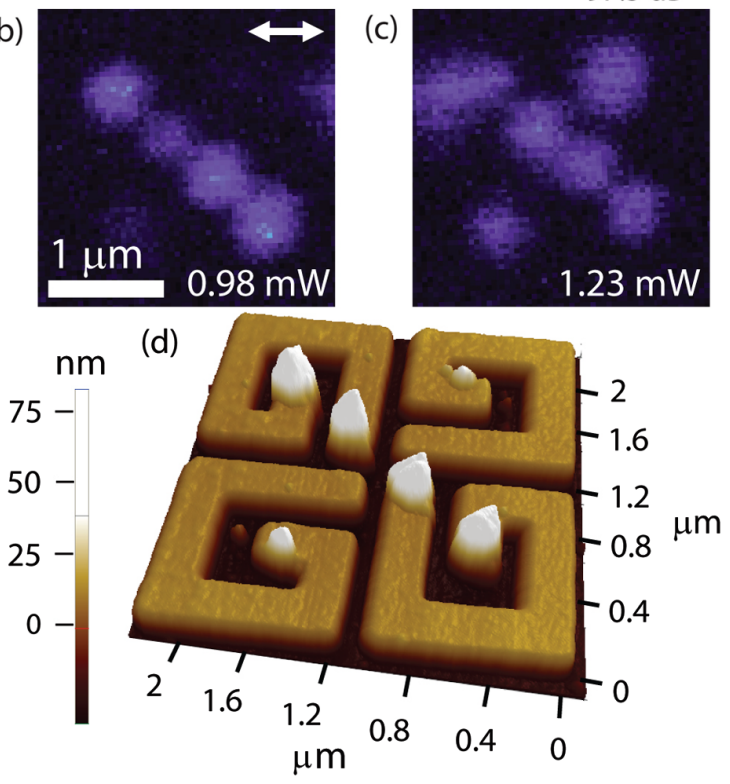

FIG. 2 (color online). Better mapping: higher laser power reveals additional features of the plasmonic pattern. (a) Theoretical simulation of the electric currents in the nickel nanostructures. The highest electric field enhancements are indicated with full white circles. The simulation also reveals that there are two other regions with high field enhancement, indicated with dashed white circles. (b) For a laser power of $0.98 \mathrm{~mW}$, SHG microscopy shows the highest field enhancements. The white arrow indicates the direction of the incident light polarization. Upon increasing the laser power to $1.23 \mathrm{~mW}$ (c), the SHG microscopy also reveals the enhancements within the dashed white circles. Although these additional hotspots are well resolved in terms of shape and contrast, they are rather large. Consequently, pinpointing the plasmon excitation on the nanostructures remains a challenge. However, upon examining the imprint on the structures with AFM (d), the regions of field enhancements are very well resolved and correspond perfectly to the simulation results.

This agreement validates our method, which, moreover, is not restricted to nickel.

We have investigated the possibility to imprint local field enhancements on the surface of palladium. Figure 3(a) shows an AFM picture of the unit cell of the nanostructured array under investigation. As it is often the case, some resist that remained after the lift-off procedure can be seen on the surface of the unit cell. This unit cell is the mirror image of the one that was used for nickel. Just as with nickel, SHG microscopy reveals that there are local
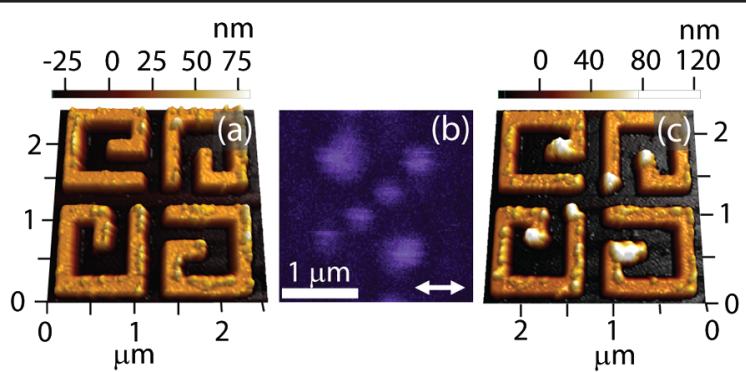

FIG. 3 (color online). Hotspot decoration beyond nickel: palladium. An AFM image of the nonilluminated unit cell of the palladium nanostructures array (a). It is a mirror image of the unit cell used for nickel. (b) SHG microscopy of the palladium nanostructures reveals the local field enhancements. The white arrow indicates the direction of the incident light polarization. Following the illumination, the hotspots are imprinted on the palladium nanostructures as evidenced by the AFM image (c).

field enhancements. Indeed, Fig. 3(b) shows SHG hotspots that are very similar to those in nickel, only mirrored. Furthermore, the AFM image in Fig. 3(c) shows that these hotspots are imprinted on the palladium surface. So far, we have demonstrated that the technique is applicable to both nickel and palladium nanostructures that are $G$ shaped; what about other geometries?

Figure 4(a) presents the geometry and dimensions of a different array of nanostructures. Each element is a nanobrick made of nickel: $200 \mathrm{~nm}$ wide and $40 \mathrm{~nm}$ thick. The length starts at $200 \mathrm{~nm}$ and increases in steps of $50 \mathrm{~nm}$. As can be seen in Fig. 4(b), these structures give rise to local field enhancements. For a length of $850 \mathrm{~nm}$, there are

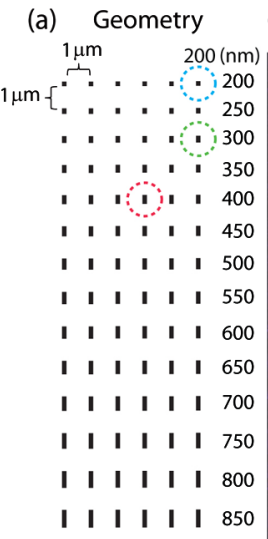

(b) SHG

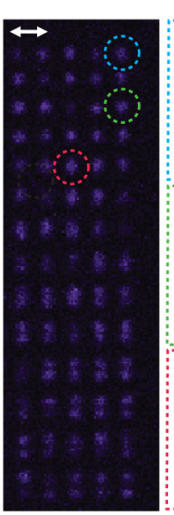

(c) Simulation

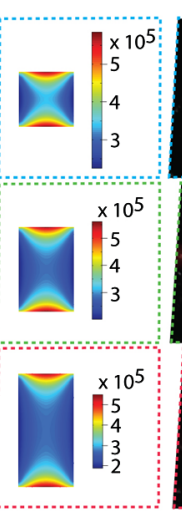

(d) AFM

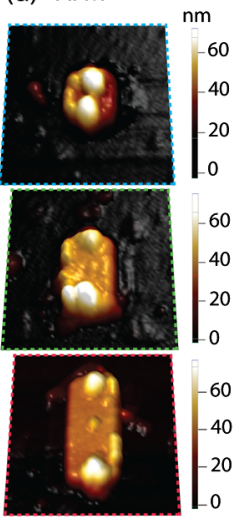

FIG. 4 (color online). Hotspot decoration beyond the $G$-shaped geometry: nanobricks. (a) An array of nickel nanobricks (rectangular hexahedrons), $40 \mathrm{~nm}$ thick, with increasing length in steps of $50 \mathrm{~nm}$. (b) Under the direction of light polarization, indicated with the white arrow, two local field enhancements form on the edges of each structure. SHG microscopy starts to resolve these field enhancements for structure lengths of approximately $650 \mathrm{~nm}$. (c) Numerical simulations of the electric currents in the three encircled nanostructures indicate that there are two hotspots at the edges. (d) AFM images demonstrate that the plasmon imprinting perfectly resolves the local field enhancements even in the smallest structures. 
two clearly distinguishable SHG hotspots at the edges of the structures. However, below $650 \mathrm{~nm}$, it becomes difficult to separate these two hotspots due to limitations of the optical resolution. Nevertheless, numerical simulations of the nanobricks indicate that there are two hotspots at the edges of even the smallest structures; see Fig. 4(c). With better resolution, these should be experimentally observable. And indeed, upon examination of the hotspot decorations with AFM, see Fig. 4(d), the regions of local field enhancements can be perfectly resolved, down to the smallest structures. Size wise, resolution is ultimately limited by the fact that hotspot decoration consumes material from the structures themselves. We believe that laserinduced atomic desorption plays an important role in the process.

Laser-induced atomic desorption is due to the collective excitations of the surface electrons under the influence of the incident electromagnetic field. Such desorption based on surface-plasmon excitations has been reported in both nanoparticles [18] and thin films [19]. Additionally, the role of plasmons in the desorption process was confirmed theoretically [20]. Following desorption, the atoms are deposited in the regions of local field enhancement. The exact mechanism is likely akin to surface-plasmon optical tweezers, with hotspots acting as optical traps [21,22]. The whole process results in structural modifications far above the atomic scale and has been reported before [23]. However, the process does not occur in all metals.

It should be noted that we have not observed any plasmonic hotspot decorations on nanostructures made of gold, although $G$-shaped nanostructures of gold exhibit plasmon related hotspots [15]. At $800 \mathrm{~nm}$, plasmons can propagate over $\sim 50 \mu \mathrm{m}$ in $\mathrm{Au}$, over $\sim 2 \mu \mathrm{m}$ in Ni, and over $\sim 3 \mu \mathrm{m}$ in $\mathrm{Pd}$. The plasmon propagation length is limited by the Ohmic losses of the electrons, which cause heating of the material. This heating is clearly much larger in $\mathrm{Ni}$ and $\mathrm{Pd}$ than in Au. Furthermore, $\mathrm{Ni}$ and $\mathrm{Pd}$ have relatively close melting temperatures: $1455^{\circ} \mathrm{C}$ and $1554^{\circ} \mathrm{C}$, respectively. The role of Ohmic losses is also evidenced by the fact that increasing laser power reveals additional hotspots in Fig. 2. Consequently, for the plasmonic hotspot decoration method reported in this Letter, plasmonic materials with high loss are of interest. In the case of materials with less loss, such as gold or silver, the loss could perhaps be provided by the dielectric layer. For instance, based on the similar aspects between hotspot decoration mapping and photoinduced migration in polymers[24,25], we believe that such polymers could contribute to the imprinting.

In conclusion, we reported hotspot decoration: a very user-friendly and robust technique for imaging plasmonic patterns in metal nanostructures with high resolution. Our findings open a new direction for mapping the plasmonic patterns in nanostructures with surface probing techniques. In future studies, smaller structures and different materials should be investigated. Besides its obvious use as a high-resolution imaging tool, our technique can also find applications as a writing method. For instance, it could be used in preparing tips for scanning-probe microscopy. The oxidation of $\mathrm{Ni}$ at the hotspots indicates that the plasmonic maxima act as chemical nanoreactors. Other chemical reactions and processes could benefit from these nanoreactors, for example, selective polymerization that is triggered by the strong local electric fields.

We thank Niels Verellen for the Pd deposition. We acknowledge financial support from the Fund for scientific research Flanders (FWO-V), the K.U. Leuven (GOA), Methusalem Funding by the Flemish government and the Belgian Inter-University Attraction Poles IAP Programmes. V. K. V., W. G., and A. V. S. are grateful for the support from the FWO-Vlaanderen. O. A. A. is partly supported by the Russian Foundation for Basic Research. B. DC. is thankful to the IWT.

*Corresponding author. v.k.valev@fys.kuleuven.be; http://www.valev.org

[1] R. D. Smith, J. B. Pendry, and M. C. K. Wiltshire, Science 305, 788 (2004).

[2] D. Schurig et al., Science 314, 977 (2006).

[3] N. Engheta, A. Salandrino, and A. Alù, Phys. Rev. Lett. 95, 095504 (2005).

[4] N. Engheta, Science 317, 1698 (2007).

[5] C. Walther, G. Scalari, M. Ines Amanti, M. Beck, and J. Faist, Science 327, 1495 (2010).

[6] E. J.R. Vesseur, R. de Waele, M. Kuttge, and A. Polman, Nano Lett. 7, 2843 (2007).

[7] J. Nelayah et al., Nature Phys. 3, 348 (2007).

[8] R. Hillenbrand, F. Keilmann, P. Hanarp, D. S. Sutherland, and J. Aizpurua, Appl. Phys. Lett. 83, 368 (2003).

[9] R. Esteban et al., Nano Lett. 8, 3155 (2008).

[10] R. Olmon et al., Phys. Rev. Lett. 105, 167403 (2010).

[11] M. Farsari, M. Vamvakaki, and B. N. Chichkov, J. Opt. 12, 124001 (2010).

[12] V. K. Valev et al., Nano Lett. 9, 3945 (2009).

[13] V. K. Valev et al., ACS Nano 5, 91 (2011).

[14] V. K. Valev et al., Opt. Mater. Express 1, 36 (2011).

[15] V. K. Valev et al., Opt. Express 18, 8286 (2010).

[16] Y. Schols and G. A.E. Vandenbosch, IEEE Trans. Antennas Propag. 55, 1086 (2007).

[17] M. Vrancken and G. A.E. Vandenbosch, IEE Proc.Microw. Antennas Propag., 149, 265 (2002).

[18] W. Hoheisel, K. Jungmann, M. Vollmer, R. Weidenauer, and F. Trager, Phys. Rev. Lett. 60, 1649 (1988).

[19] I. Lee, T. A. Callcott, and E. T. D. Arakawa, Phys. Rev. B 47, 6661 (1993).

[20] R. Monreal and S. P. Apell, Phys. Rev. B 41, 7852 (1990).

[21] M. Righini, G. Volpe, C. Girard, D. Petrov, and R. Quidant, Phys. Rev. Lett. 100, 186804 (2008).

[22] A. N. Grigorenko, N. W. Roberts, M. R. Dickinson, and Y. Zhang, Nat. Photon. 2, 365 (2008).

[23] M. Vollmer, R. Weidenauer, W. Hoheisel, U. Schulte, and F. Trager, Phys. Rev. B 40, 12509 (1989).

[24] Y. Gilbert et al., Opt. Express 13, 3619 (2005).

[25] Y. Gilbert et al., Opt. Lett. 31, 613 (2006). 